\title{
Human pericardial proteoglycan 4 (lubricin): Implications for postcardiotomy intrathoracic adhesion formation
}

Daniel S. J. Park, MSc, ${ }^{a}$ Suresh C. Regmi, PhD, ${ }^{b}$ Daniyil A. Svystonyuk, BSc, ${ }^{a}$ Guoqi Teng, PhD, ${ }^{a}$

Darrell Belke, PhD, ${ }^{\mathrm{a}}$ Jeannine Turnbull, BSc, ${ }^{\mathrm{a}}$ David G. Guzzardi, BSc, ${ }^{\text {a }}$ Sean Kang, BSc, ${ }^{\mathrm{a}}$ Mary K. Cowman, PhD, ${ }^{\mathrm{c}}$ Tannin A. Schmidt, PhD, ${ }^{\text {b,d }}$ and Paul W. M. Fedak, MD, PhD, FRCSC

\begin{abstract}
Objective: Intrapericardial fibrous adhesions increase the risk of sternal reentry. Proteoglycan 4/lubricin (PRG4) is a mucin-like glycoprotein that lubricates tissue compartments and prevents inflammation. We characterized PRG4 expression in human pericardium and examined its effects in vitro on human cardiac myofibroblast fibrotic activity and in vivo as a measure of its therapeutic potential to prevent adhesions.
\end{abstract}

Methods: Full-length PRG4 expression was determined using Western blot analysis and amplified luminescent proximity homogeneous assay in human pericardial tissues obtained at cardiotomy. The in vitro effects of PRG4 were investigated on human cardiac myofibroblasts for cell adhesion, collagen gel contraction, and cell-mediated extracellular matrix remodeling. The influence of PRG4 on pericardial homeostasis was determined in a chronic porcine animal model.

Results: PRG4 is expressed in human pericardial fluid and colocalized with pericardial mesothelial cells. Recombinant human PRG4 prevented human cardiac myofibroblast attachment and reduced myofibroblast activity assessed using collagen gel contraction assay $(64.6 \% \pm 8.1 \%$ vs $47.1 \% \pm 6.8 \% ; P=.02)$. Using a microgel assay, human cardiac myofibroblast mediated collagen fiber remodeling was attenuated by PRG4 ( $1.17 \pm 0.03$ vs $0.90 \pm 0.05 ; P=.002)$. In vivo, removal of pericardial fluid alone induced severe intrapericardial adhesion formation, tissue thickening, and inflammatory fluid collections. Restoration of intrapericardial PRG4 was protective against fibrous adhesions and preserved the pericardial space.

Conclusions: For the first time, we show that PRG4 is expressed in human pericardial fluid and regulates local fibrotic myofibroblast activity. Loss of PRG4enriched pericardial fluid after cardiotomy might induce adhesion formation. Therapeutic restoration of intrapericardial PRG4 might prevent fibrous/inflammatory adhesions and reduce the risk of sternal reentry. (J Thorac Cardiovasc Surg 2018;156:1598-608)

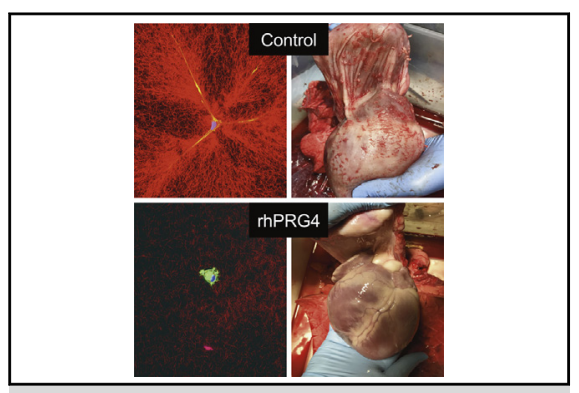

rhPRG4 attenuates human fibroblast fibrotic activity and prevents pericardial adhesions.

\section{Central Message}

Full-length PRG4 is documented in human pericardial fluid and shown in vitro to regulate human myofibroblast activity. In vivo, rhPRG4 prevented intrapericardial fibrous adhesion formation.

\section{Perspective}

Postoperative intrapericardial adhesions increase reoperative risk. We show that PRG4 is expressed in human pericardial fluid and regulates human fibroblast activity. Removal of pericardial fluid induced severe adhesion formation whereas PRG4 replacement was protective. Restoring intrapericardial PRG4 after cardiac surgery might attenuate postoperative adhesions and increase the safety of reoperation.

See Editorial Commentary page 1609.
From the ${ }^{\text {a Section }}$ of Cardiac Surgery, Department of Cardiac Sciences, Cumming School of Medicine, Libin Cardiovascular Institute of Alberta, ${ }^{b}$ Faculty of Kinesiology, University of Calgary, Calgary, Alberta, Canada; ${ }^{\mathrm{c} D e p a r t m e n t}$ of Chemical and Biomolecular Engineering, New York University Tandon School of Engineering, New York, NY; and ${ }^{\mathrm{d} B i o m e d i c a l ~ E n g i n e e r i n g ~ D e p a r t m e n t, ~ S c h o o l ~}$ of Dental Medicine, University of Connecticut Health Center, Farmington, Conn.

Support for this work was provided to P.W.M.F. and T.A.S. by a Canadian Institutes of Health Research Project Bridge Grant and the Canada Research Chairs Program. D.S.J.P. is funded by the Canadian Institutes of Health Research, Alberta Innovates-Health Solutions and Cumming School of Medicine, University of Calgary.

Received for publication Sept 21, 2017; revisions received March 30, 2018; accepted for publication March 30, 2018; available ahead of print May 30, 2018.

Address for reprints: Paul W. M. Fedak, MD, PhD, FRCSC, C880, 1403-29 St NW,

Calgary, Alberta T2N 2T9, Canada (E-mail: paul.fedak@gmail.com).

$0022-5223 / \$ 36.00$

Copyright (c) 2018 by The American Association for Thoracic Surgery

https://doi.org/10.1016/j.jtcvs.2018.03.170
Postoperative retrosternal pericardial adhesion formation is a consequence of inflammatory and fibrotic responses after tissue injury. Adhesions are associated with greater mortality risks and risk of injury for patients who undergo sternal reentry. ${ }^{1}$ Previously reported complications include damage to previous bypass grafts, the heart, and innominate vessels,

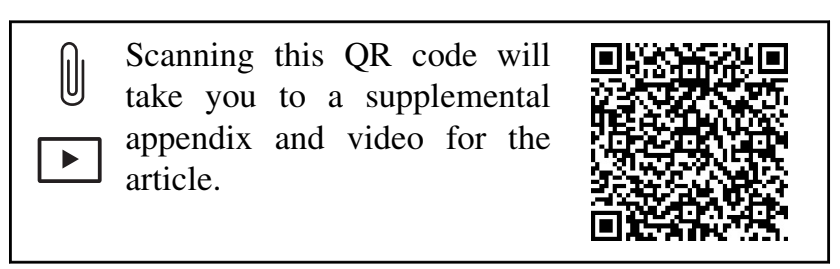




\section{Abbreviations and Acronyms \\ AlphaLISA $=$ amplified luminescent proximity homogeneous assay \\ DAPI $=4^{\prime}, 6$-diamidino-2-phenylindole \\ $\mathrm{ECM}=$ extracellular matrix \\ $\mathrm{mAb} \quad=$ monoclonal antibody \\ PBS $=$ phosphate buffered saline \\ PRG4 = proteoglycan 4/lubricin \\ rhPRG4 = recombinant human proteoglycan 4 (lubricin) \\ SDS-PAGE $=$ sodium dodecyl sulfate polyacrylamide gel electrophoresis \\ SMA $=$ smooth muscle actin \\ TGF $=$ transforming growth factor}

among others. ${ }^{2}$ Despite these well documented risks, there is no widely accepted and validated therapy in contemporary surgical practice to prevent or attenuate postsurgical intrapericardial adhesion formation.

During cardiac surgery, denudation of the pericardial mesothelium from serous pericardial layers creates a pathologic condition facilitating the formation of a fibrin mesh network. ${ }^{3}$ Fibrin mesh creates a platform by which proinflammatory and profibrotic cytokines, such as transforming growth factor- $\beta 1$ (TGF- $\beta 1$ ), can increase myofibroblast migration and activation. ${ }^{4,5}$ Increased $\alpha$-smooth muscle actin $(\alpha$-SMA) protein expression in myofibroblasts has been shown in surgical adhesions suggesting increased cellular activity and a profibrotic response. ${ }^{6}$ Myofibroblast activation and extracellular matrix (ECM) protein production and remodeling are important mechanisms by which mild adhesions transition to dense, fibrous adhesion requiring sharp dissection during sternal reentry. ${ }^{3,6,7}$

Lubricin, also known as proteoglycan 4 (PRG4), is a mucinous glycoprotein found endogenously in numerous tissue compartments with diverse biological functions, of which joint synovial fluid is the best known example. ${ }^{8,9}$ Lubricin/PRG4 has anti-inflammatory properties and has been shown to influence fibroblast adhesion and proliferation. ${ }^{10-13}$ Administration of recombinant human PRG4 (rhPRG4) in a rat model of intra-abdominal adhesions was shown to limit the development of postoperative adhesions. ${ }^{14} \mathrm{We}$ and others speculate that loss of PRG4 might induce adhesion formation and accordingly, restoring lost PRG4 within tissue compartments might help prevent postsurgical adhesion formation.

PRG4 expression in the human pericardial mesothelium tissue was recently documented through immunohistochemistry; however, expression of full-length PRG4 has not been confirmed. ${ }^{15}$ We therefore sought to further explore expression of PRG4 in human pericardial tissues as well as its fluid. In addition, we explored the biological effects of rhPRG4 on human cardiac myofibroblast activity and cell-mediated ECM remodeling in vitro. Furthermore, we assessed the in vivo effects of pericardial fluid loss and its restoration with rhPRG4 in a preclinical porcine model to provide proof-of-concept data.

\section{METHODS}

\section{Recombinant Human Lubricin/PRG4}

Purified rhPRG4 solution was obtained from Lubris BioPharma, LLC (Framingham, Mass). The rhPRG4 was generated and purified as described previously. $^{10-12}$

\section{Human Tissue Procurement}

Pericardial fluid and tissue samples were obtained from consenting patients undergoing cardiac surgery at Foothills Medical Centre (Calgary, Alberta, Canada). Experiments involving human tissue usage were approved by the Conjoint Health Research Ethics Board at the University of Calgary and conform to the Declaration of Helsinki.

\section{Western Blot Analysis}

Western blot analysis to detect PRG4 protein in pericardial fluid samples were performed as previously described. ${ }^{16}$ Briefly, samples were subjected to Novex 3-8\% Tris-Acetate sodium dodecyl sulfate polyacrylamide gel electrophoresis (SDS-PAGE). Blotted membranes were incubated overnight with anti-PRG4 monoclonal antibody (mAb) 9G3 (EMD Millipore, Billerica, Mass) at 1:50,000 (20 ng/mL), washed, then treated with secondary antibody goat anti-mouse horseradish peroxidase (SigmaAldrich, St Louis, Mo) at 1:1000. Immunoreactive bands were detected using enhanced chemiluminescence substrate (SuperSignal West Femto; ThermoScientific, Waltham, Mass) and a gel imaging system (ImageQuant LAS 4000; GE, Toronto, Ontario, Canada).

\section{Amplified Luminescent Proximity Homogeneous Assay Quantification of Full-Length PRG4}

PRG4 was quantitatively determined using amplified luminescent proximity homogeneous assay (AlphaLISA; Perkin Elmer, Boston, Mass) technology. Briefly, streptavidin modified donor beads were bound to biotin-labeled rhPRG4, and protein G modified acceptor beads were bound to anti-PRG4 mAb 9G3. Competitive inhibition of the rhPRG4-9G3 interaction by free unlabeled PRG4 in solution was used for PRG4 quantification (Appendix E1).

\section{Immunohistochemistry}

Pericardial tissue samples were harvested from cardiac surgery patients and fixed in 4\% paraformaldehyde for 15 minutes. ${ }^{17}$ After incubation in mAb 9G3 at 1:200 dilution in 1.5\% goat serum phosphate buffered saline (PBS) overnight at $4{ }^{\circ} \mathrm{C}$, sections were washed in PBS and incubated with Alexa-Fluor 594 rhodamine-conjugated goat anti-mouse immunoglobulin G secondary antibody (Life Technologies, Carlsbad, Calif) at a dilution of 1:100. Sections were mounted with 4',6-diamidino-2-phenylindole (DAPI) stain (Vectashield; Vector Laboratories, Burlingame, Calif). Samples were imaged using confocal microscopy.

\section{Human Cardiac Myofibroblast Cell Isolation}

As previously described, right atrial appendage biopsy samples were rinsed in phosphate buffered saline (PBS; Lonza, Walkersville, MD). ${ }^{18,19}$ Tissues were minced and explant cultured with Iscove's Modified Dulbecco's medium (IMDM) supplemented with 10\% fetal bovine serum (Gibco Life Technologies, Burlington, Ontario, Canada) and 5\% penicillin-streptomycin (Life Technologies, Burlington, Ontario, Canada). 
Cells were grown at $37^{\circ} \mathrm{C}$ in $5 \% \mathrm{CO}_{2}$ and passaged when cells reached $90 \%$ confluency. Only cells from passages 2 through 5 were used.

\section{Assessment of Cell Adhesion to Coated Surfaces}

Cells were seeded on 24-well culture plates either noncoated or coated with $200 \mu \mathrm{L}$ PBS with or without $100 \mu \mathrm{g} / \mathrm{mL}$ rhPRG4 for 24 hours at a concentration of 10,000 cells $/ \mathrm{mL}$ at $1 \mathrm{~mL} /$ well. Cells were imaged qualitatively for adherence to coated culture plates 24 hours after seeding and again 48 hours after cell seeding after a PBS wash.

\section{3-D Collagen Gel Contraction Assay}

The 3-D collagen gel contraction assay was performed as previously described. ${ }^{18,19}$ IMDM alone (serum free medium control; $500 \mu \mathrm{L}$ ) or containing $10 \mathrm{ng} / \mathrm{mL}$ human recombinant TGF- $\beta 1$ (Gibco Life Technologies) was added into each well, with or without $100 \mu \mathrm{g} / \mathrm{mL}$ rhPRG4. Images of the collagen matrix gel surface area were obtained 48 hours from the time of release from the side of the wells. Percent contraction was measured using ImageJ software (National Institutes of Health, Bethesda, Md).

\section{Annexin V/Propidium Iodide Cell Apoptosis Assay}

As previously described, cells isolated from collagen matrices, resuspended in $200 \mu \mathrm{L}$ of cold binding buffer, were stained with annexin $\mathrm{V}$ and propidium iodide (BD Pharmingen, Becton Dickinson, San Jose, Calif) according to the manufacturer's instructions. ${ }^{18,19}$ Percentages of annexin $\mathrm{V}$ and propidium iodide positive cells were measured using flow cytometry as a measure of early apoptosis and late apoptosis/necrosis.

\section{Assessment of Cell Morphology and ECM Remodeling}

As previously described, individual human cardiac myofibroblasts were seeded in a 3-D type I collagen matrix polymerized on a noncontractile, inert nylon grid with $1500 \mu \mathrm{m} \times 1500 \mu \mathrm{m}$ dimensions. ${ }^{19}$ Type I bovine dermal collagen (Advanced Biomatrix, San Diego, Calif) was diluted to $1.0 \mathrm{mg} / \mathrm{mL}$ at $\mathrm{pH} 7.4$ using $\mathrm{PBS}$ and $0.1 \mathrm{~N} \mathrm{NaOH}$, respectively. Polymerized collagen grids were removed from culture dishes using PBS and inserted to serum-free IMDM with $10 \mathrm{ng} / \mathrm{mL}$ human recombinant TGF- $\beta 1$, with or without $100 \mu \mathrm{g} / \mathrm{mL}$ rhPRG4. Cells were seeded at approximately 1200 cells per grid and treated for 24 hours. Cells were fixed in $4 \%$ PFA, permeabilized using $0.1 \%$ Triton $\mathrm{X}$ and stained with AlexaFluor 488 phalloidin (Life Technologies) at 1:100 dilution, rhodamine tagged 9G3 and DAPI at 1:100 dilution. Cell roundness as a measure of

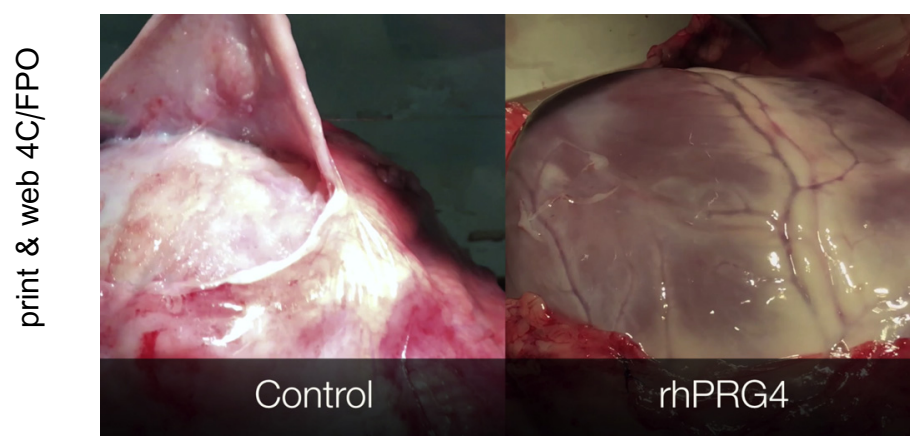

VIDEO 1. Description of porcine model and postmortem qualitative analysis of intrapericardial adhesion severity between groups (control vs rhPRG4) at 10 days. Video available at: https://www.jtcvs.org/article/ S0022-5223(18)31028-6/fulltext. cell morphology was made using the multicell outliner plug-in for ImageJ. Values approaching 1 are increasingly round. Collagen fiber alignment was measured as an indication of local ECM remodeling. Six analyses per cell, selected randomly, at a minimum of 6 cells per patient were made. Collagen fiber alignment was calculated from the intensity curve obtained by ImageJ as the area under the curve at the peak intensity value $\pm 10^{\circ}$, with larger values indicating greater fiber alignment.

\section{Preclinical Porcine Pericardial Adhesion Model}

A preclinical model was developed using $30 \mathrm{~kg}$ farm swine (York $\times$ Landrace; Britestone Farming Co, Alberta, Canada). The study was approved by the Institutional Animal Care Committee and performed in accordance with Canadian Council on Animal Care. Animals were intubated, mechanically ventilated and anesthesia maintained with continuous infusions of isoflurane, oxygen, alfaxalone, and dexmedetomidine. After anterolateral thoracotomy, a pericardial incision was made and pericardial fluid removed (Video 1). Pericardial fluid was either not replaced (control group: $\mathrm{n}=2$ ) or replaced with $10 \mathrm{~mL}$ of $1.24 \mathrm{mg} / \mathrm{mL}$ rhPRG4 in PBS (rhPRG4 group: $\mathrm{n}=2$ ). Pericardium was sealed with a purse string suture, chest closed, and animals recovered. Ten days postoperatively, at postmortem exam pericardial fluid was collected and the extent and tenacity of intrapericardial adhesion and images recorded were qualitatively assessed.

\section{Statistics}

One-way analysis of variance was performed using Bonferroni's multiple comparisons test, $P<.05$, for experiments with 3 comparison groups. Unpaired $t$ tests were performed for experiments with 2 comparison groups.

\section{RESULTS}

\section{Full-Length PRG4 Protein Is Expressed in Human Pericardial Fluid as Well as Tissues}

Human pericardial fluid samples were tested for the presence of full-length PRG4 using Western blot analysis (Figure 1, A) and further quantified using AlphaLISA $(\mathrm{n}=16$, Figure $1, B)$. Tissue histology of serous pericardium showed areas of intact pericardial mesothelial layer (Figure $1, C$ ) and areas of damaged pericardial mesothelial layers (Figure 1,D). Expression of full-length PRG4 was limited to the pericardial mesothelial cells lining the pericardial cavity (Figure 1, $E$ and $F$ ).

\section{rhPRG4 Prevents Human Cardiac Myofibroblast Adhesion to Coated Surfaces}

Human cardiac myofibroblasts were seeded onto tissue culture plates either uncoated or coated with PBS with or without $100 \mu \mathrm{g} / \mathrm{mL}$ rhPRG4 to test antiadhesive effects of rhPRG4. Human cardiac myofibroblasts seeded on noncoated or PBS coated tissue culture plates adhered to the plates as shown by the stellate morphology of the cells and clear cell extensions (Figure 2). Cardiac myofibroblasts seeded onto rhPRG4 coated culture plates were completely prevented from adhering to the plate surface as shown by the free-floating cells with round cell morphology (Figure 2). The lack of adhesion was confirmed by washing the plates, which removed all cells in the rhPRG4 coated group but not the other groups (Figure 2). 


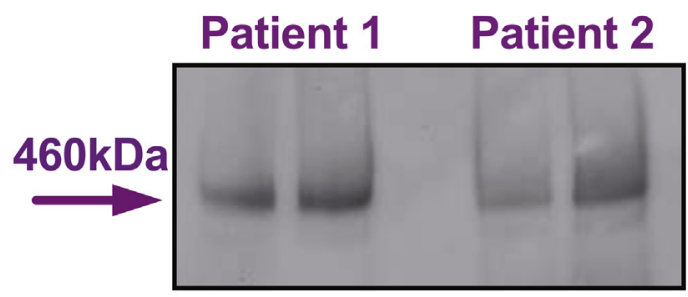

A
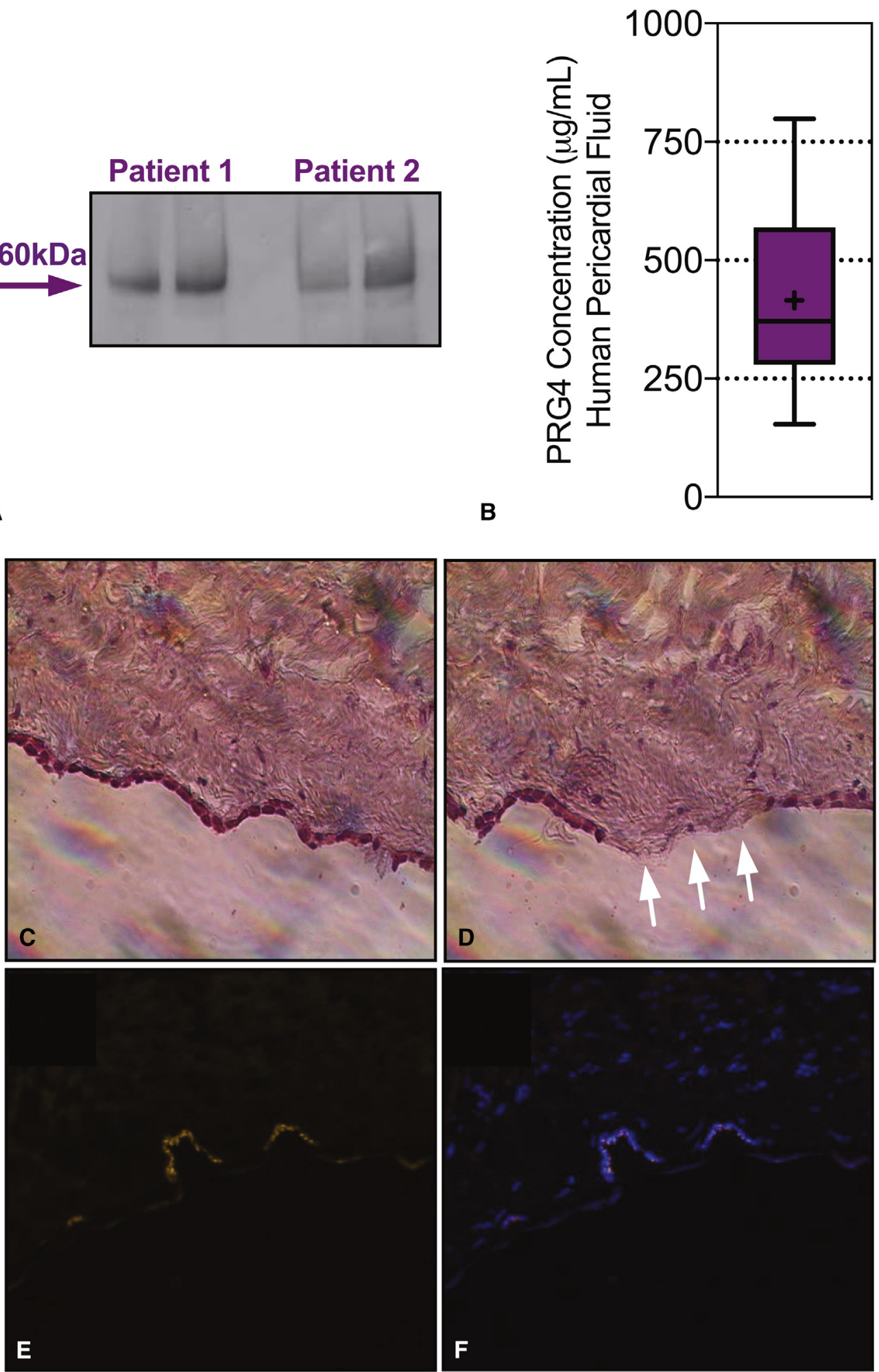

FIGURE 1. A, Western blot using anti-proteoglycan 4/lubricin (PRG4) monoclonal antibody 9G3 showing expression of full-length PRG4 protein in human pericardial fluid (approximately $460 \mathrm{kDa}$ ). B, Box and whisker plot showing quantitative assessment of full-length PRG4 in human pericardial fluid using amplified luminescent proximity homogeneous assay $(\mathrm{N}=16)$. Minimum and maximum values shown with bar indicating median and + indicating mean value. Human pericardial tissue hematoxylin and eosin histology showing (C) an intact pericardial mesothelial cell layer and (D) a damaged pericardial mesothelium. E, Fluorescence imaging via confocal microscopy of PRG4 detected in the pericardial mesothelium via rhodamine tagged anti-PRG4 monoclonal antibody 9G3. F, Superimposed image of 4',6-diamidino-2-phenylindole (nuclei) stain with PRG4 detection. 


\section{Pre-Wash}

\section{Post-Wash}
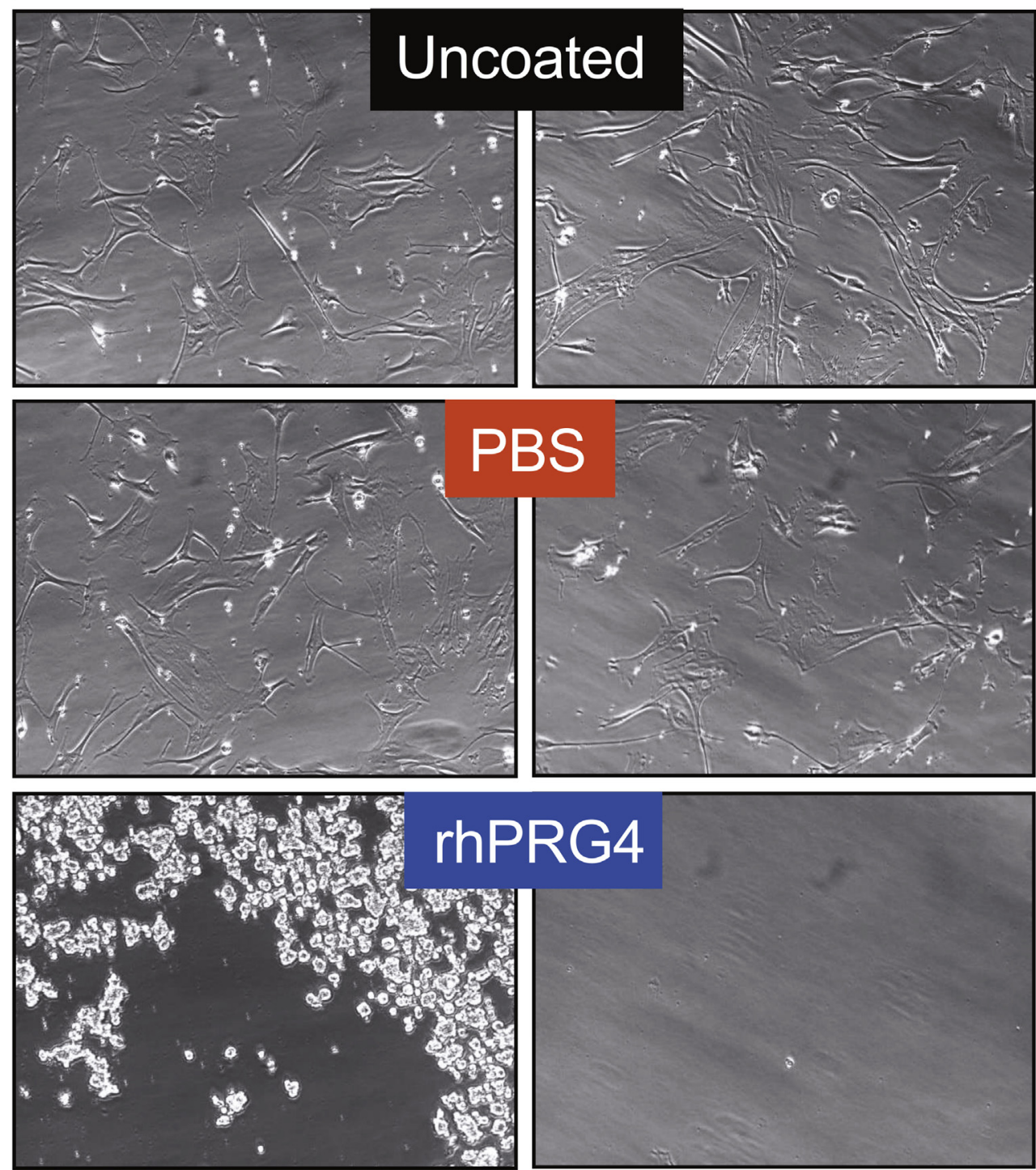

FIGURE 2. Cardiac myofibroblasts imaged 24 hours after being seeded on tissue culture plates that were either left uncoated, coated with phosphate buffered saline $(P B S)$ or coated with rhPRG4. Images on the left side show cells before washing and images on the right side show effects of washing. rhPRG4, Recombinant human proteoglycan 4 (lubricin).

\section{rhPRG4 Attenuates Cell-Mediated Collagen Matrix Gel Contraction}

The bioactive effect of rhPRG4 on the attenuation of human cardiac myofibroblast mediated collagen matrix gel contraction was measured. Collagen gel contraction is a well established model of human wound healing by fibrous scar tissue formation. Human cardiac myofibroblasts embedded in collagen matrices produced a baseline level of contraction and the addition of TGF- $\beta 1$ stimulated contraction (Figure 3,A). In the presence of TGF- $\beta 1$, the addition of rhPRG4 attenuated collagen matrix gel contraction $(64.6 \% \pm 8.1 \%$ for TGF- $\beta 1$ vs $47.1 \% \pm 6.8 \%$ for TGF- $\beta 1$ with rhPRG4; $\mathrm{n}=4$ patients; $P=.02$; Figure $3, B)$. Expression of $\alpha$-SMA protein was assessed as a measure of myofibroblast activation. In the presence of TGF- $\beta 1$, rhPRG 4 did not alter $\alpha$-SMA expression (mean fluorescence intensity normalized to baseline: $1.35 \pm 0.65$ for TGF- $\beta 1$ vs $1.28 \pm 0.67$ for TGF- $\beta 1$ with 
rhPRG4; $\mathrm{n}=4$ patients, $P=.89$; Figure $3, C$ ). This observation suggests that the attenuation of gel contraction was not due to a shift in the cell phenotype toward an inactivated and quiescent noncontractile fibroblast phenotype.

\section{rhPRG4 Does Not Induce Human Cardiac Myofibroblast Cell Injury or Death}

Annexin V and propidium iodide dye-based apoptosis and necrosis assays were performed using fluorescence to confirm that the observed attenuation of collagen matrix gel contraction was not due to cell toxicity from rhPRG4 exposure. There were no significant differences in the percentage of cells that were detected as dead or in the early stages of apoptosis in the rhPRG4 treated group compared with controls (Figure 4).

\section{rhPRG4 Attenuates Cell-Mediated ECM \\ Remodeling}

Local collagen fiber alignment was analyzed as a measure of cell-mediated ECM remodeling. TGF- $\beta 1$ was used to stimulate human cardiac myofibroblasts to contract local collagen fibers (compaction) and remodel collagen fibers toward an increased alignment (Figure 5, A). The addition of rhPRG4 significantly decreased local cell-mediated ECM remodeling (collagen fiber alignment index: $1.17 \pm 0.03$ for control vs $0.90 \pm 0.05$ for rhPRG4; $\mathrm{n}=3$ patients; $P=.002$; Figure $5, B$ ). Cell roundness was analyzed as a measure of cell morphology. The addition of TGF- $\beta 1$ induced human cardiac myofibroblasts to assume a stellate morphology with long cell extensions consistent with active ECM remodeling (Figure 5, A). The addition of rhPRG4 attenuated cell morphology toward increased roundness and decreased number of cell extensions (cell roundness index: $0.60 \pm 0.09$ for controls vs $0.84 \pm 0.06$ for rhPRG4; $\mathrm{n}=3$ patients; $P=.02$; Figure $5, C$ ) suggesting a reduced capacity for local cell-mediated ECM remodeling. Rhodamine-labeled rhPRG4 was used to determine that rhPRG4 localized at the cell surface of a human cardiac myofibroblast (Figure 6) and did not appear to be internalized within the cell.

\section{rhPRG4 Preserves Pericardial Integrity and Prevents Adhesion Formation}

Using a preclinical porcine model $(n=4)$, minimally invasive removal of pericardial fluid from the pericardial space resulted in marked intrapericardial adhesion formation, epi- and pericardial thickening, and inflammatory serosanguinous fluid accumulation $(20-30 \mathrm{~mL}) 10$ days later (Figure 7 and Video 1). In contrast, restoration of pericardial fluid with rhPRG4-enriched PBS solution completely prevented adhesion formation and epicardial or pericardial thickening. Recovered pericardial fluid $(6-12 \mathrm{~mL})$ was straw colored and consistent with normal healthy pericardial fluid. All animals recovered well and there was no evidence of impaired wound healing in either group.

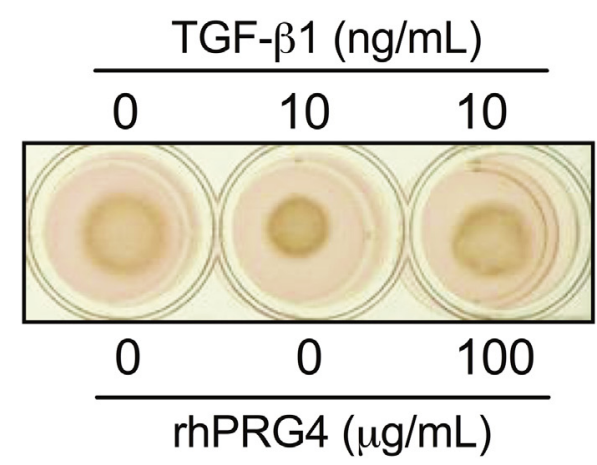

A

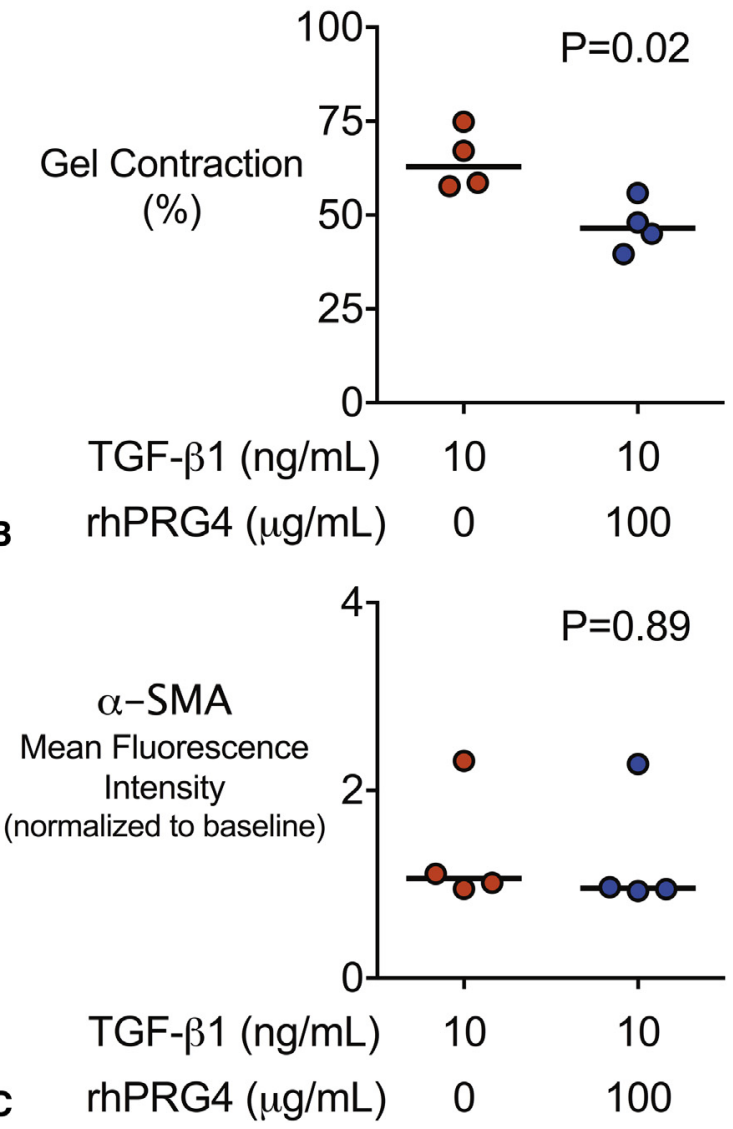

FIGURE 3. A, Representative images of human cardiac myofibroblasts embedded in a 3-D collagen matrix gel contraction model. B, Contraction percentage compared with baseline contraction. Bar indicates group median. C, Alpha-smooth muscle actin $(\alpha-S M A)$ protein expression on the basis of mean fluorescence intensity detection via flow cytometry analysis. Bar indicates group median. TGF, Transforming growth factor; rhPRG4, recombinant human proteoglycan 4 (lubricin).

\section{DISCUSSION}

Postsurgical adhesion formation is believed to involve similar processes regardless of the tissue in question, including fibrin mesh network formation, increases in proinflammatory mediators, and myofibroblast migration with local ECM remodeling. ${ }^{3}$ More recently, the usage of antiadhesive agents to fibroblasts and inhibitors 

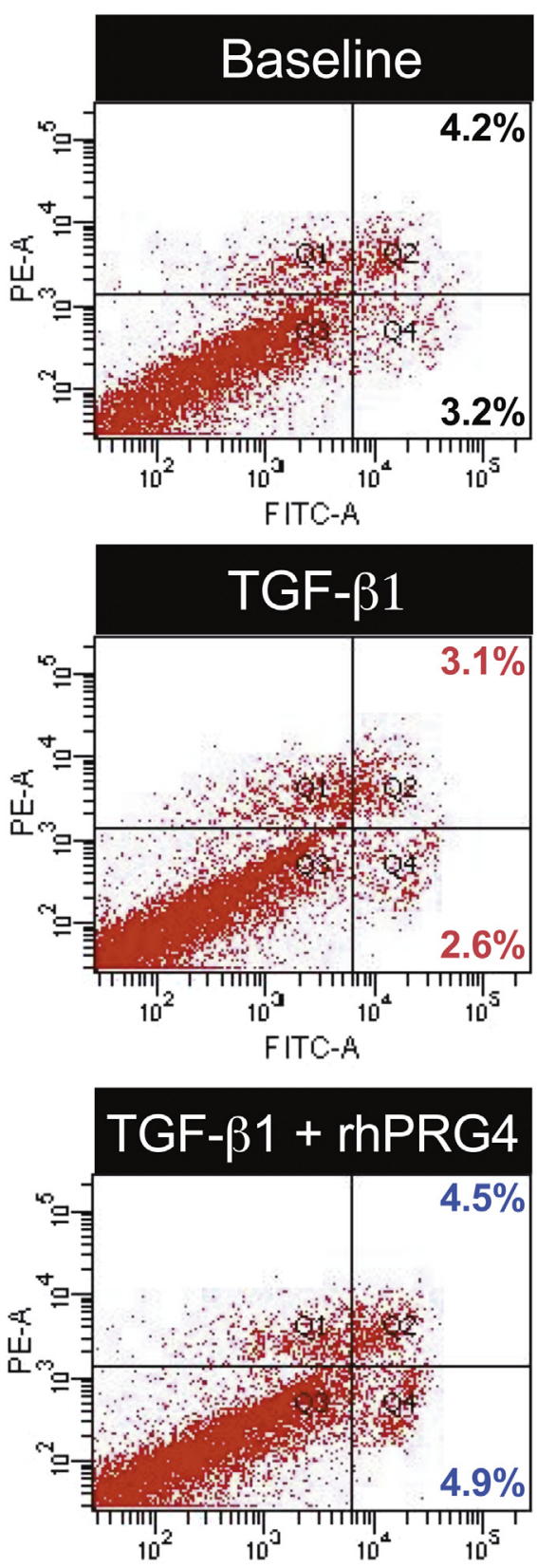

A

FITC-A
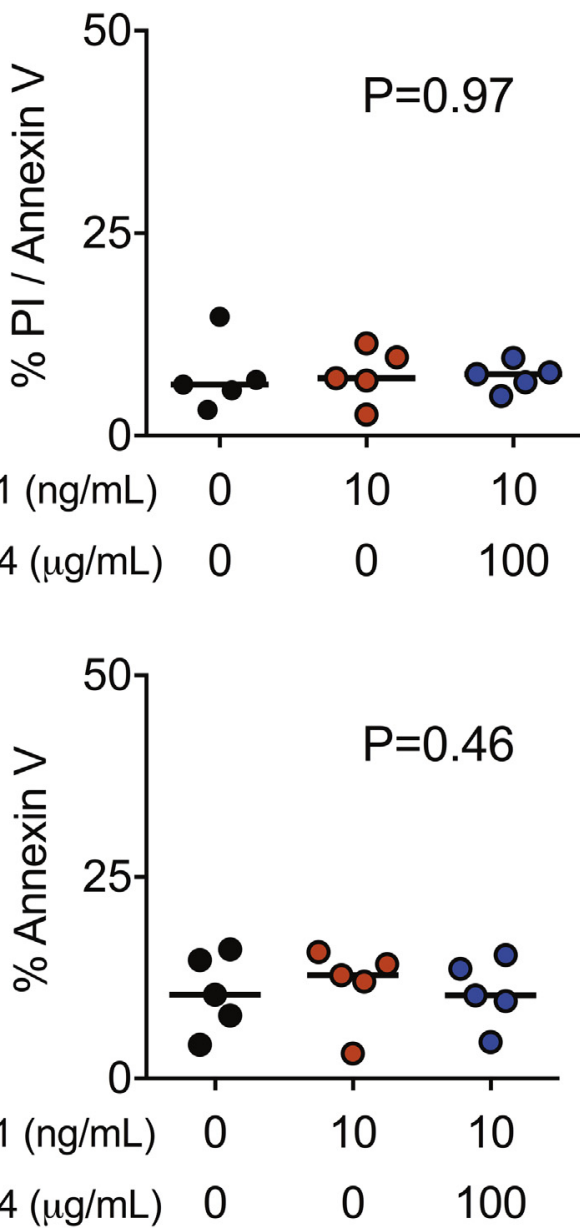

FIGURE 4. A, Representative fluorescence measurement of fluorescein isothiocyanate (FITC)-conjugated annexin V and propidium iodide (PI) staining of human cardiac myofibroblasts. Percentage of annexin V and phycoerythrin $(P E)$-conjugated PI positive cells as a measure of (B) late apoptosis/necrosis or (C) early apoptosis. Bar indicates group median. $T G F$, Transforming growth factor; rhPRG4, recombinant human proteoglycan 4 (lubricin).

of inflammatory mediators have been shown to reduce postoperative pericardial adhesions in animal models. ${ }^{5,20-23}$ Blockage of the TGF- $\beta 1$ signaling pathway through administration of anti-inflammatory and antifibrotic peptide, ghrelin, in a mouse model reduced postoperative adhesion severity by increasing the expression of inhibitory proteins Smad6/7 in the TGF- $\beta 1$ signaling pathway. ${ }^{23}$ Antiadhesive agents have also been studied as promising therapeutics to limit postoperative pericardial adhesions after cardiac surgery. Antiadhesive chitosan derivative, N,O-carboxymethyl chitosan, applied on injured surfaces after cardiac surgical injury in a rabbit model decreased severity of postoperative adhesions. ${ }^{20}$ In vitro study of N,O-carboxymethyl chitosan has shown that it can prevent adhesion of fibroblasts and macrophages to precoated surfaces suggesting a biophysical barrier mechanism of preventing adhesion. These studies collectively suggest that therapeutic agents targeting 

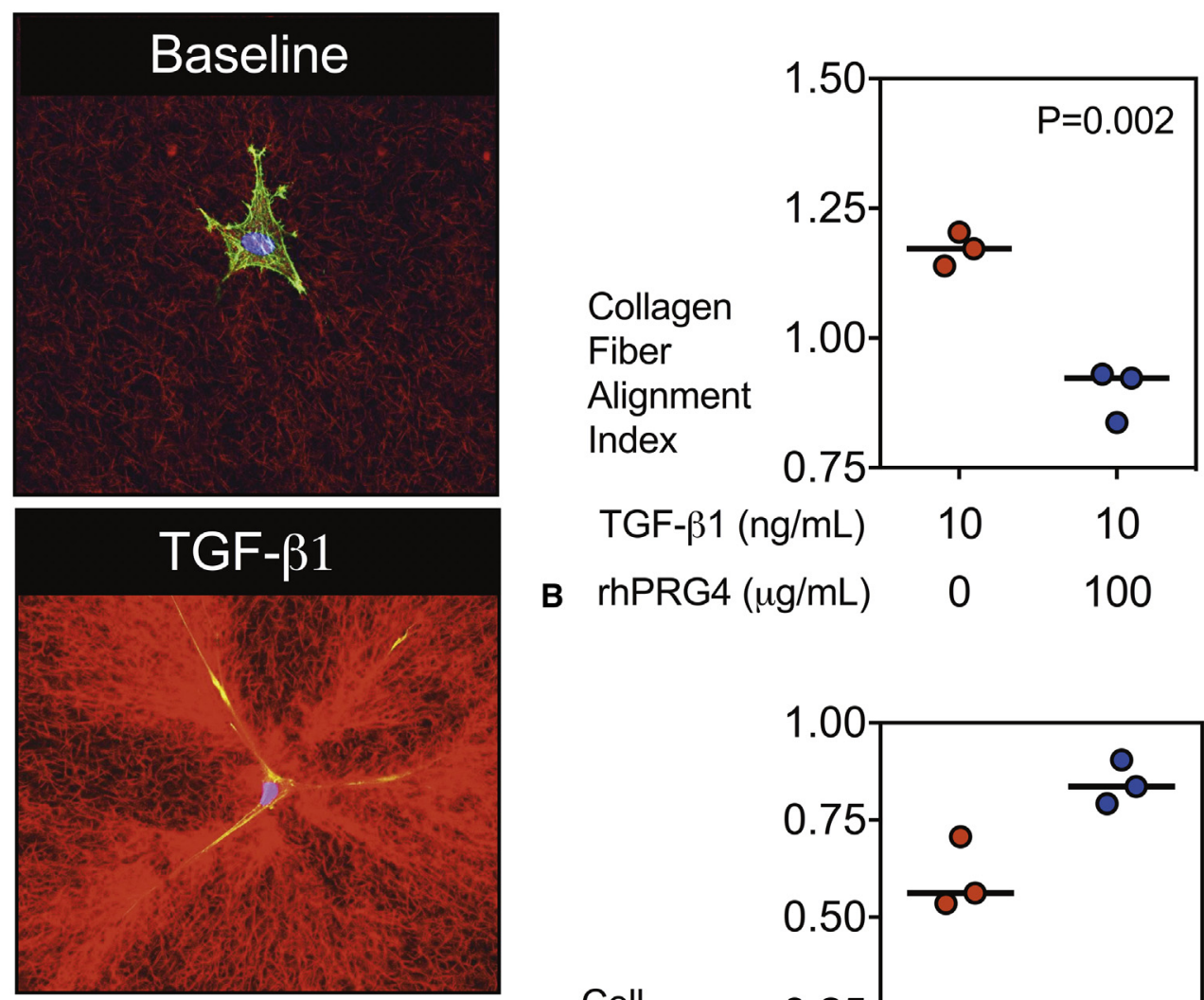

B $\mathrm{rhPRG} 4(\mu \mathrm{g} / \mathrm{mL}) \quad 0 \quad 100$

\section{TGF- $\beta 1+$ rhPRG4}

\section{A}

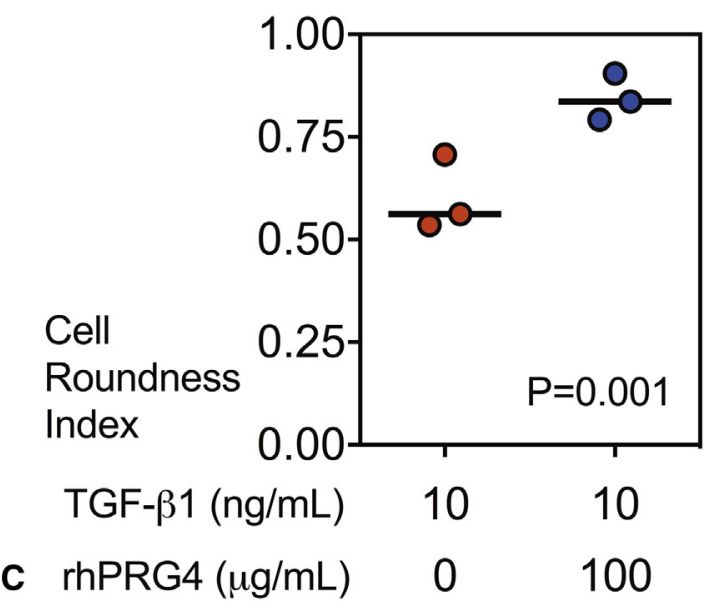

FIGURE 5. A, Human cardiac myofibroblasts embedded in a 3-D collagen matrix imaged via confocal microscopy; F-actin, green phalloidin stain; nuclei, blue 4',6-diamidino-2-phenylindole; collagen, red auto reflectance. B, Collagen fiber alignment index as a measure of matrix remodeling. Bar indicates group median. C, Cell circularity as a morphological measure of myofibroblast activation. Bar indicates group median. TGF, Transforming growth factor; rhPRG4, recombinant human proteoglycan 4 (lubricin).

myofibroblasts might be effective in limiting postoperative pericardial adhesion formation.

rhPRG4 as a therapeutic agent has been tested in human applications, such as for dry eyes, and does not induce adverse events. ${ }^{24}$ The lubricating ability of PRG4 has been connected to the O-linked glycosylation of the mucin domain. ${ }^{25}$ Further, PRG4 can also elicit anti-inflammatory responses by binding to cell receptors, such as CD44 and toll-like receptors 2 and $4 .{ }^{10-12}$ rhPRG4, as applied in an animal model of abdominal adhesion, has been shown to reduce adhesion tenacity and measures of fibrosis and inflammation. ${ }^{14}$ The mechanisms by which rhPRG4 is able to limit surgical adhesion development is not known. This study uniquely investigated the expression of PRG4 protein in human pericardial fluid and tissue and the in vitro effects of rhPRG4 on human cardiac myofibroblast adhesion, activation, morphology, and local ECM remodeling.

To our knowledge, this study is the first to quantify fulllength PRG4 in human pericardial fluid. PRG4 expressed by pericardial mesothelial cells lining the pericardial cavity might be released into the pericardial cavity. ${ }^{15}$ Mesothelial 


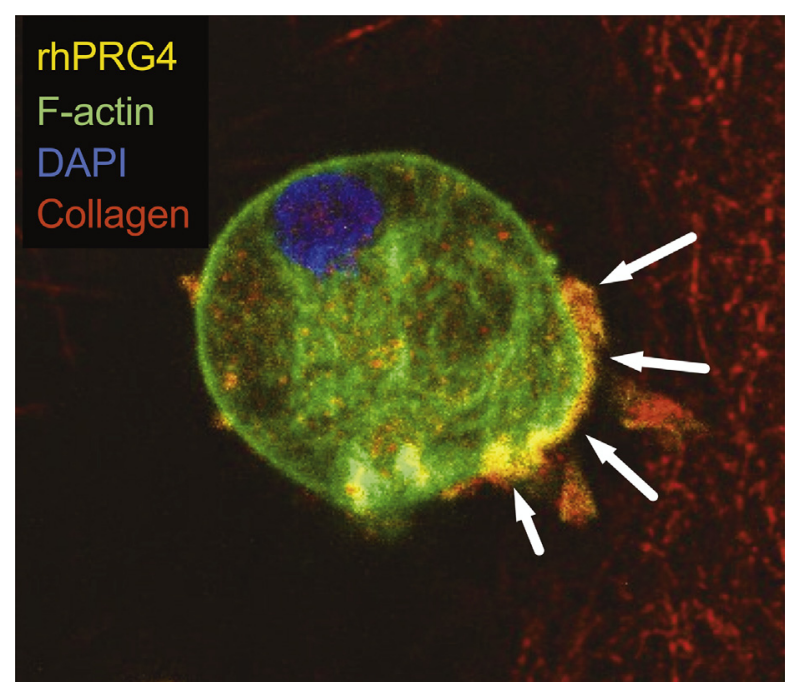

FIGURE 6. Human cardiac myofibroblast embedded in a 3-D collagen matrix imaged via confocal microscopy; F-actin, green phalloidin stain; nuclei, blue DAPI; collagen, red auto reflectance; full-length rhPRG4, yellow rhodamine tagged fluorescence. Arrows, rhodamine-tagged rhPRG4 localized at the cell surface. $r h P R G 4$, Recombinant human proteoglycan 4 (lubricin); DAPI, 4',6-Diamidino-2-phenylindole.

cells are also present in pericardial fluid and this cell population cannot be ruled out as a possible source of PRG4 in the pericardial fluid. ${ }^{26}$ The lubricating function of pericardial fluid might in part be explained by the presence of PRG4.

In addition to expression, we explored the biological effects of PRG4. The ability of rhPRG4 coated surfaces to prevent adhesion of human cardiac myofibroblasts might be explained in part by the antiadhesive properties of the mucin domain of PRG4. However, studies suggest that the mucin domain might not be solely responsible for its important antiadhesive properties. ${ }^{13}$ It is unclear how rhPRG4 influences cardiac myofibroblast activity, however we speculate that it might act on cell ECM receptors to prevent myofibroblast-mediated collagen synthesis and fiber compaction (scar formation and wound contraction).

rhPRG4 was able to attenuate TGF- $\beta 1$ mediated collagen matrix gel contraction by human cardiac myofibroblasts. rhPRG4 did not induce toxic effects on human cardiac myofibroblasts supporting that the observed attenuation response was not due to cell death or injury. Interestingly, rhPRG4 did not decrease $\alpha$-SMA expression. Our data suggest that rhPRG4 might not alter the myofibroblast phenotype but might exert its effects by preventing interactions of human cardiac myofibroblasts with local ECM. rhPRG4 decreased local ECM remodeling. The change in cell morphology of rhPRG4-treated cardiac myofibroblasts might also be explained by the decrease in cell matrix interaction. We also show that rhPRG4 targets the cell surface and in so doing, might act on cell receptors and prevent cell matrix interactions.

\section{Clinical Significance}

Postoperative retrosternal and intrapericardial adhesions pose a significant risk for cardiac surgery patients undergoing reentry. ${ }^{1}$ We provide novel data that suggest PRG4 plays an important role in maintaining pericardial homeostasis. Removal of pericardial fluid after opening the pericardium during cardiac surgery will result in loss of PRG4 in the pericardial space, which might promote adhesion development. As a proof of concept, we modeled pericardial fluid removal (control) and the effects of PRG4 restoration. Removal of pericardial fluid resulted in severe adhesion formation and inflammation. These novel data highlight a protective role of pericardial fluid in maintaining the integrity of the pericardial space. We observed that restoration of PRG4 prevented fibrous adhesions, tissue thickening, and inflammatory fluid accumulation. Replacement of pericardial fluid with rhPRG4 alone rescued the pericardial space and restored normal pericardial health. Beyond effects on attenuating myofibroblast activity, the putative mechanisms underlying this observed beneficial effect remain to be determined.

rhPRG4 has already been used via topical administration in a clinical trial for dry eye disease and was shown to be a safe as well as effective treatment. ${ }^{24}$ This clinical study leveraged basic research showing the expression of PRG4 by corneal and conjunctival epithelial cells at the ocular surface and its function as a boundary lubricant molecule to reduce friction between the cornea-eyelid interface. ${ }^{9}$ In light of the findings of the present study, showing presence as well as in vitro function of PRG4 relevant to pericardial tissue, coupled with the availability of clinical-grade rhPRG4 for study already shown to be effective in another application, we believe rhPRG4 restoration into the pericardial cavity might be highly feasible. Replacing and restoring lost pericardial fluid with a rhPRG4-rich solution through intrapericardial injection or closing the pericardium with an rhPRG4-enhanced biomaterial might be a suitable therapeutic to prevent postsurgical pericardial adhesion development.

\section{Limitation}

The in vitro doses of rhPRG4 used in this study were on the basis of physiological concentrations of PRG4 found in synovial fluid whereas the in vivo dose was approximately 2- to 3-fold higher than the human pericardial levels observed (for the first time) in our study. ${ }^{27}$ Our in vivo findings, although profound, are preliminary and will require further validation with more comprehensive testing with longer follow-up intervals, altered doses, and additional control groups. Safety end points and specific effects on wound healing need further evaluation. The 

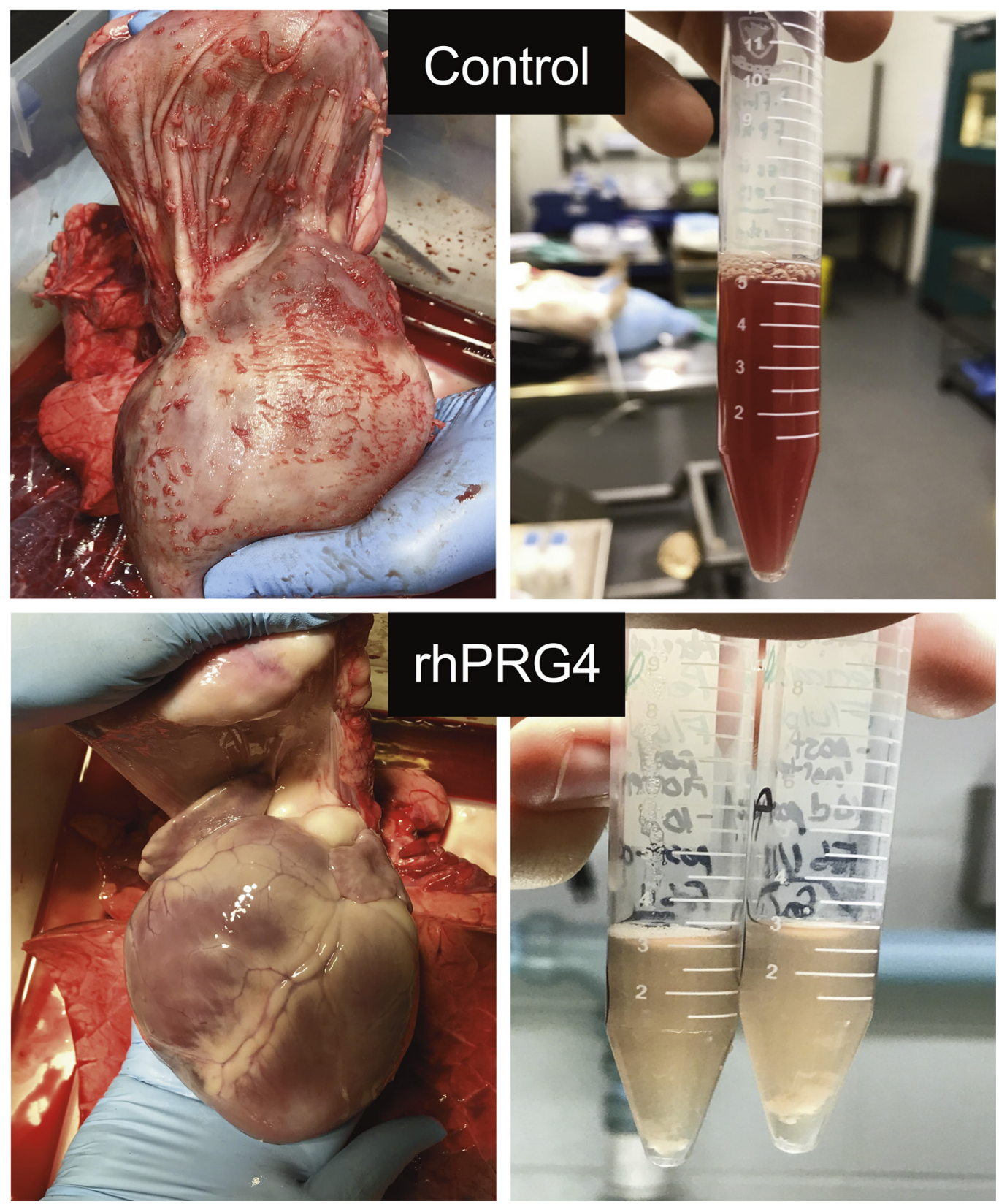

FIGURE 7. In a porcine model, removal of pericardial fluid resulted in a marked development of intrapericardial adhesions, diffuse epi- and pericardial thickening, and accumulation of serosanguinous fluid consistent with a heightened inflammatory response. Restoring intrapericardial PRG4 prevented intrapericardial adhesion formation, tissue thickening, and inflammation as compared with the control group. rhPRG4, Recombinant human proteoglycan 4 (lubricin).

efficacy of rhPRG4 will need to be determined in a surgical model that more robustly mimics critical stimuli of adhesion formation including local trauma, retained blood, and the inflammatory effects of cardiopulmonary bypass. We aim to address these important questions in our future work.

\section{CONCLUSIONS}

Full-length PRG4 protein is expressed in human pericardial fluid and the mesothelium lining the pericardial cavity. rhPRG4 decreases human cardiac myofibroblast-mediated local ECM remodeling, an important phase of surgical adhesion development. Restoration of lost intrapericardial PRG4 is shown to prevent adhesions and tissue inflammation in a preclinical animal model. rhPRG4 should be investigated as a therapeutic agent to prevent or attenuate postsurgical pericardial adhesions.

\section{Conflict of Interest Statement}

Dr Schmidt holds patents on rhPRG4, is a paid consultant for Lubris BioPharma, LLC, and holds equity in Lubris 
BioPharma. All other authors have nothing to disclose with regard to commercial support.

\section{References}

1. Park CB, Suri RM, Burkhart HM, Greason KL, Dearani JA, Schaff HV, et al Identifying patients at particular risk of injury during repeat sternotomy: analysis of 2555 cardiac reoperations. J Thorac Cardiovasc Surg. 2010;140:1028-35.

2. Roselli EE, Pettersson GB, Blackstone EH, Brizzio ME, Houghtaling PL, Hauck R, et al. Adverse events during reoperative cardiac surgery: frequency, characterization, and rescue. J Thorac Cardiovasc Surg. 2008;135:316-23.

3. Cannata A, Petrella D, Russo CF, Bruschi G, Fratto P, Gambacorta M, et al. Postsurgical intrapericardial adhesions: mechanisms of formation and prevention. Ann Thorac Surg. 2013;95:1818-26.

4. Williams RS, Rossi AM, Chegini N, Schultz G. Effect of transforming growth factor beta on postoperative adhesion formation and intact peritoneum. J Surg Res. 1992;52:65-70.

5. Gorvy DA, Herrick SE, Shah M, Ferguson MW. Experimental manipulation of transforming growth factor-beta isoforms significantly affects adhesion formation in a murine surgical model. Am J Pathol. 2005;167:1005-19.

6. Saed GM, Diamond MP. Differential expression of alpha smooth muscle cell actin in human fibroblasts isolated from intraperitoneal adhesions and normal peritoneal tissues. Fertil Steril. 2004;82:1188-92.

7. Bayhan Z, Zeren S, Kocak FE, Kocak C, Akcilar R, Kargi E, et al. Antiadhesive and anti-inflammatory effects of pirfenidone in postoperative intra-abdominal adhesion in an experimental rat model. J Surg Res. 2016;201:348-55.

8. Rhee DK, Marcelino J, Baker M, Gong Y, Smits P, Lefebvre V, et al. The secreted glycoprotein lubricin protects cartilage surfaces and inhibits synovial cell overgrowth. J Clin Invest. 2005;115:622-31.

9. Schmidt TA, Sullivan DA, Knop E, Richards SM, Knop N, Liu S, et al. Transcription, translation, and function of lubricin, a boundary lubricant, at the ocular surface. JAMA Ophthalmol. 2013;131:766-76.

10. Iqbal SM, Leonard C, Regmi SC, De Rantere D, Tailor P, Ren G, et al. Lubricin/ proteoglycan 4 binds to and regulates the activity of toll-like receptors in vitro. Sci Rep. 2016;6:18910

11. Alquraini A, Garguilo S, D'Souza G, Zhang LX, Schmidt TA, Jay GD, et al. The interaction of lubricin/proteoglycan 4 (PRG4) with toll-like receptors 2 and 4: an anti-inflammatory role of PRG4 in synovial fluid. Arthritis Res Ther. 2015;17: 353.

12. Al-Sharif A, Jamal M, Zhang LX, Larson K, Schmidt TA, Jay GD, et al. Lubricin/ proteoglycan 4 binding to CD44 receptor: a mechanism of the suppression of proinflammatory cytokine-induced synoviocyte proliferation by lubricin. Arthritis Rheumatol. 2015:67:1503-13.

13. Aninwene GE II, Yang Z, Ravi V, Jay GD, Webster TJ. Lubricin as a novel nanostructured protein coating to reduce fibroblast density. Int J Nanomedicine. 2014; 9:3131-5.
14. Oh J, Kuan KG, Tiong LU, Trochsler MI, Jay G, Schmidt TA, et al. Recombinant human lubricin for prevention of postoperative intra-abdominal adhesions in a rat model. J Surg Res. 2017;208:20-5.

15. Solka KA, Miller IJ, Schmid TM. Sialidase unmasks mucin domain epitopes of lubricin. J Histochem Cytochem. 2016;64:647-68.

16. Steele BL, Alvarez-Veronesi MC, Schmidt TA. Molecular weight characterization of PRG4 proteins using multi-angle laser light scattering (MALLS). Osteoarthritis Cartilage. 2013;21:498-504.

17. Abubacker S, Dorosz SG, Ponjevic D, Jay GD, Matyas JR, Schmidt TA Full-length recombinant human proteoglycan 4 interacts with hyaluronan to provide cartilage boundary lubrication. Ann Biomed Eng. 2016;44 1128-37.

18. Park D, Mewhort H, Teng G, Belke D, Turnbull J, Svystonyuk D, et al. Heparin augmentation enhances bioactive properties of acellular extracellular matrix scaffold. Tissue Eng Part A. 2018;24:128-34.

19. Teng G, Svystonyuk D, Mewhort HE, Turnbull JD, Belke DD, Duff HJ, et al. Tetrandrine reverses human cardiac myofibroblast activation and myocardial fibrosis. Am J Physiol Heart Circ Physiol. 2015;308:H1564-74.

20. Zhou J, Liwski RS, Elson C, Lee TD. Reduction in postsurgica adhesion formation after cardiac surgery in a rabbit model using N,O-carboxymethyl chitosan to block cell adherence. J Thorac Cardiovasc Surg. 2008;135:777-83.

21. Soga Y, Takai S, Koyama T, Okamoto Y, Ikeda T, Nishimura K, et al. Attenuation of adhesion formation after cardiac surgery with a chymase inhibitor in a hamster model. J Thorac Cardiovasc Surg. 2004;127:72-8.

22. Okamoto Y, Takai S, Yamada M, Miyazaki M. Chymase inhibitors may prevent postoperative adhesion formation. Fertil Steril. 2002;77:1044-8.

23. Bianchi E, Boekelheide K, Sigman M, Lamb DJ, Hall SJ, Hwang K. Ghrelin inhibits post-operative adhesions via blockage of TGF- $\beta$ signaling pathway. PLos One. 2016;11:e0153968.

24. Lambiase A, Sullivan BD, Schmidt TA, Sullivan DA, Jay GD, Truitt ER III, et al A two-week, randomized, double-masked study to evaluate safety and efficacy of lubricin $(150 \mu \mathrm{g} / \mathrm{mL})$ eye drops versus sodium hyaluronate (HA) $0.18 \%$ eye drops (Vismed $($ ) in patients with moderate dry eye disease. Ocul Surf. 2017; 15:77-87.

25. Jay GD, Harris DA, Cha CJ. Boundary lubrication by lubricin is mediated by O-linked beta(1-3)Gal-GalNAc oligosaccharides. Glycoconj J. 2001;18: 807-15.

26. Vogiatzidis K, Zarogiannis SG, Aidonidis I, Solenov EI, Molyvdas PA, Gourgoulianis KI, et al. Physiology of pericardial fluid production and drainage. Front Physiol. 2015;6:62.

27. Schmidt TA, Gastelum NS, Nguyen QT, Schumacher BL, Sah RL. Boundary lubrication of articular cartilage: role of synovial fluid constituents. Arthritis Rheum. 2007;56:882-91

Key Words: ibroblasts, surgical adhesions, proteoglycan 4 


\section{APPENDIX E1. AMPLIFIED LUMINESCENT PROXIMITY HOMOGENEOUS ASSAY ANALYSIS}

PRG4 was quantitatively determined using AlphaLISA; Perkin Elmer. ${ }^{\mathrm{E} 1, \mathrm{E} 2}$ Full-length rhPRG4, obtained from Lubris BioPharma, LLC, ${ }^{\mathrm{E} 3, \mathrm{E} 4}$ was biotinylated (brhPRG4) using a commercially available kit (EZ-Link Sulfo-NHS-LC-Biotinylation Kit, ThermoScientific), as per the manufacturer's instructions. The protein integrity of brhPRG4 was confirmed using SDS-PAGE protein stain and Western blot analysis with $\mathrm{mAb} 9 \mathrm{G} 3{ }^{\mathrm{E} 5}$ and biotinylation was confirmed using Western blot analysis with streptavidin-peroxidase. Then brhPRG4 was mixed with either rhPRG4, to generate a control curve, or the pericardial fluid sample. Specifically, $5 \mu \mathrm{L}$ of brhPRG4 (at $8 \mathrm{ng} / \mathrm{mL}$ ) was incubated with $5 \mu \mathrm{L}$ of rhPRG4 (at concentrations of $0.8,8,80,240,800,8000,80,000 \mu \mathrm{g} / \mathrm{mL}$ ), $5 \mu \mathrm{L}$ of pericardial fluid sample at multiple serial dilutions in opaque 96-well half plates (Perkin Elmer) for $30 \mathrm{mi}-$ nutes. Anti-PRG4 mAb 9G3 (10 $\mu \mathrm{L}$; Millipore) at $1000 \mathrm{ng} / \mathrm{mL}$ was then added to the reaction volume and allowed to incubate for 2 hours. Next, $10 \mu \mathrm{L}$ of protein Gcoated AlphaLISA acceptor beads (Perkin Elmer) at $80 \mu \mathrm{g} / \mathrm{mL}$ was added to the reaction volume and allowed to incubate for 1 hour. Last, $10 \mu \mathrm{L}$ of streptavidin coated AlphaScreen donor beads (Perkin Elmer) at $80 \mu \mathrm{g} / \mathrm{mL}$ were added to the reaction volume, under low light, and allowed to incubate for 1 hour (with the plate protected from light). The total reaction volume summed to $40 \mu \mathrm{L}$, including $5 \mu \mathrm{L}$ of sample, with final concentrations of $1 \mathrm{ng} / \mathrm{mL}, 500 \mathrm{ng} / \mathrm{mL}, 20 \mu \mathrm{g} / \mathrm{mL}$, and $20 \mu \mathrm{g} / \mathrm{mL}$ for the brhPRG4, mAb 9G3, G-protein coated acceptor beads, and streptavidin coated donor beads, respectively. Plates were then read on a Molecular Devices (Sunnyvale, Calif) SpectraMax i3 using an excitation wavelength of $680 \mathrm{~nm}$ and emission wavelength of $625 \mathrm{~nm}$. All reagents were prepared and/or diluted in PBS with $0.05 \%$ Tween 20, and all incubations were done at room temperature, with gentle nutation, and using a plate sealer to prevent evaporation. All samples were run in duplicate.

With the method described, competitive inhibition of the rhPRG4-9G3 interaction by free unlabeled PRG4 in solution was used for PRG4 quantification. Because mAb 9G3 recognizes the mucin domain of full-length PRG4, ${ }^{\mathrm{E}, \mathrm{E} 6}$ the method developed using full-length rhPRG4 detects as well as quantifies full-length PRG4 in samples of interest. Indeed, the 9G3 immunoreactive species observed in the human pericardial fluid samples were full-length with the expected apparent molecular weight of approximately $460 \mathrm{kDa}$ on the $3-8 \%$ Tris-Acetate SDS-PAGE Western blots (Figure 1, A). The mucin domain is functionally determinant for full-length PRG4, as it is necessary for boundary lubricating function ${ }^{\mathrm{E} 7}$ and it has also been reported to affect PRG4's interaction with CD44. ${ }^{\text {E8 }}$

\section{E-References}

E1. Ullman EF, Kirakossian H, Singh S, Wu ZP, Irvin BR, Pease JS, et al. Luminescent oxygen channeling immunoassay: measurement of particle binding kinetics by chemiluminescence. Proc Natl Acad Sci U S A. 1994;91:5426-30.

E2. Huang X, Schmidt TA, Shortt C, Arora S, Asari A, Kirsch T, et al. A competitive alphascreen assay for detection of hyaluronan. Glycobiology. 2018;28:137-47.

E3. Samsom ML, Morrison S, Masala N, Sullivan BD, Sullivan DA, Sheardown H, et al. Characterization of full-length recombinant human proteoglycan 4 as an ocular surface boundary lubricant. Exp Eye Res. 2014;127:14-9.

E4. Elsaid KA, Zhang L, Shaman Z, Patel C, Schmidt TA, Jay GD. The impact of early intra-articular administration of interleukin-1 receptor antagonist on lubricin metabolism and cartilage degeneration in an anterior cruciate ligament transection model. Osteoarthritis Cartilage. 2015;23:114-21.

E5. Abubacker S, Dorosz SG, Ponjevic D, Jay GD, Matyas JR, Schmidt TA. Full length recombinant human proteoglycan 4 interacts with hyaluronan to provide cartilage boundary lubrication. Ann Biomed Eng. 2016;44:1128-37.

E6. Ai M, Cui Y, Sy MS, Lee DM, Zhang LX, Larson KM, et al. Anti-lubricin monoclonal antibodies created using lubricin-knockout mice immunodetect lubricin in several species and in patients with healthy and diseased joints. PLoS One. 2015; 10:e0116237.

E7. Jay GD, Harris DA, Cha CJ. Boundary lubrication by lubricin is mediated by Olinked B(1-3)Gal-GalNAc oligosaccharides. Glycoconj J. 2001;18:807-15.

E8. Al-Sharif A, Jamal M, Zhang LX, Larson K, Schmidt TA, Jay GD, et al. Lubricin/proteoglycan 4 binding to CD44 receptor: a mechanism of the suppression of proinflammatory cytokine-induced synoviocyte proliferation by lubricin. Arthritis Rheumatol. 2015;67:1503-13. 